\title{
Level of VEGF-A and interleukin 6 in lacrimal fluid of patients with diabetic retinopathy
}

\author{
Christelle DOMNGANG ${ }^{1 *}$, Armelle LOWE NOUTCHIE ${ }^{1}$, Pascal DJAMEN CHUISSEU ${ }^{1}$, \\ Freddy NEMG SIMO ${ }^{2}$, BORRIS TIETCHE GALANI ${ }^{3}$, Pamela TUMCHOU MEKIEJE ${ }^{1}$, \\ Faustin Ulrich NZOUNJI ${ }^{1}$, Eric Noel DJAHMENI ${ }^{4}$, Magellan GUEWO-FOKENG ${ }^{2}$, Giles \\ $\mathrm{KAGMENI}^{4}$, Assumpta Lucienne BELLA ${ }^{4}$ and Eugene SOBNGWI ${ }^{4}$ \\ ${ }^{1}$ Department of Medicine, Medical and Biomedical Sciences, Higher Institute of Health Sciences, Université \\ des Montagnes - Bangangté. \\ ${ }^{2}$ Department of Biochemistry, Faculty of Science, Université de Yaoundé 1 - Yaoundé. \\ ${ }^{3}$ Department of Biological Sciences, Faculty of Science, University of Ngaoundéré. \\ ${ }^{4}$ Faculty of Medicine and Biomedical Science, Université de Yaoundé - Yaoundé. \\ *Corresponding author; E-mail: dockrystlnoche@gmail.com; Tel: +237 699936 709;
}

BP 208 Bangangté, Cameroun.

\begin{abstract}
Diabetic retinopathy (DR) is an ocular manifestation characterized by microvascular complications. The aim of this study was to investigate the relationship between diabetic retinopathy (DR) and two lacrimal angiogenic cytokines, interleukin-6 (Il-6) and vascular endothelial growth factor-A (VEGF-A). A cross-sectional study was conducted at Yaoundé Central Hospital from February,1 to June, 15, 2019. Twenty-six diabetic patients with retinopathy and 55 diabetics without retinopathy were enrolled. Tears were collected with Schirmer strips. The concentrations of VEGF-A and Il-6 were determined by the ELISA test. Statistical analysis was performed using Epi Data version 7.1.5.2. and GraphPad 5.0. The mean VEGF-A concentration was $163.4 \pm$ 45.1 and $149 \pm 81.8 \mathrm{pg} / \mathrm{ml}$ in patients with and without DR respectively $(\mathrm{p}=0.292)$ while tear Il-6 level was $118.5 \pm 103$ and $119 \pm 79.43 \mathrm{pg} / \mathrm{ml}$ in patients with and without DR respectively $(\mathrm{p}=0.497)$. The mean tear VEGF level was $165.2 \pm 76.10$ and $153.50 \pm 57.94 \mathrm{pg} / \mathrm{mL}$ in patients with and without diabetic maculopathy (DM) respectively $(\mathrm{p}=0.343)$ while tear Il-6 level was significantly lower in DM $(54.92 \pm 32.68)$ compared to patients without DM (159.10 \pm 89.78$)(\mathrm{p}=0.047)$. Tear Il-6 level was significantly low in patients with DM. There was no association between the 2 lacrimal biomarkers and diabetic retinopathy. Further analysis is needed for this type of screening in diabetic patients.
\end{abstract}

(C) 2020 International Formulae Group. All rights reserved.

Keywords: eye, retina, tear, diabetes, biomarker; cytokine.

\section{INTRODUCTION}

Diabetic retinopathy (DR) is the most significant ocular manifestation of diabetes and represents the most specific microvascular complication of diabetes mellitus (Cheung et al., 2010). With an estimated DR prevalence of $30 \%$ in diabetic patients, this pathology remains the main causes of blindness among diabetic people worldwide (Reutrakul et al., 2020). The global prevalence of blindness due 
to DR is around $4.8 \%$. WHO estimates that $33 \%$ of type II diabetic population will develop a retinopathy after 20 years (OMS, 2020). In Sub-Saharan Africa, this prevalence is between 15 and 52\% (Labie, 2007). According to the literature, the prevalence of diabetic retinopathy and its proliferative form is between 30.2 to $32 \%$ and 1.2 to $4.5 \%$ respectively (Burgess et al., 2013). The prevalence reported in Cameroonian studies is higher than the one reported in African studies. Koki (2010) and Jingi (2014) found a prevalence of $42 \%$ and $40 \%$ in diabetic patients respectively (Koki et al., 2010; Jingi et al. , 2014).

DR has a multifactorial and complex pathophysiology which is not well described. Previous studies found that hyperglycemia, hypertension, hyperlipidemia, and genetic background are the main systemic risk factors (Tarr et al., 2012). Inflammatory mediators, such as angiotensin I, prostaglandins, cytokines, chemokines, VEGF, and vascular cell adhesion molecule-1, have been found to be involved in macular edema pathogenesis (Ebneter and Zinkernagel, 2016). Interleukin-6 (IL-6), a pleiotropic cytokine, has been identified as the main factor of vascular endothelial factor (VEGF) activation. This agent thus causes retinal neovascularization and even hemorrhages in more advanced stages of diabetic retinopathy and may be used as a biomarker of this disease (Myśliwiec et al., 2008). An improved therapeutic approach to fight diabetic vascular complications might include blocking mechanisms of injury as well as promoting protective or regenerating factors. Less than 15 years have elapsed between the detection of high levels of VEGF in the vitreous of patients with proliferative DR and the administration of anti-VEGF in the treatment of RD diabetes (Myśliwiec et al., 2008; Ebneter and Zinkernagel, 2016).

The diagnosis of DR is based on the examination of the fundus. This contributes to the distinction of the different stages of the retinopathy and its monitoring. (Koki et al. 2010; Cheung et al., 2010) .

Ocular complications are common in type 2 diabetes in sub-Saharan Africa. There is an interest in using non-invasive and inexpensive methods to detect and/or facilitate the follow-up of diabetic retinopathy. As tears are the most accessible and less complex body fluid (than serum or plasma) and sampling is much less invasive, research is starting to focus on how disease processes affect the proteomic, lipidomic and metabolomic composition of the tear film. By determining compositional changes to tear profiles, crucial pathways in disease progression may be identified, allowing for more predictive and personalized therapy of the individual (Hagan et al., 2016) .

The study by Ang (2019) has shown that VEGF levels in tears were significantly higher amongst diabetic patients with DR compared to those without DR and were significantly associated with the severity of DR (2019). The level of Il-6 and VEGF in tears may differ according to the level of DR and compare to non-DR patient as showed by serological studies. In the best of our knowledge, the investigation of lacrimal cytokines has not been yet explored in our context. This study aims to evaluate the level of Il-6 and VEGF-A in tears among type 2 diabetes mellitus patient with and without DR.

\section{MATERIALS AND METHODS Ethical considerations}

In order to carry out the present study, we obtained an approval from the Institutional Ethical Committee of the Université des Montagnes (N²019/251/UdM/PR/CIE).

\section{Design and study setting}

We conducted an analytical, crosssectional study with a prospective recruitment, at the National Obesity Centre (CNO) of the Yaoundé's Central Hospital and Innel Medical Center for five months from February, 1 to June, 20, 2019. All biological analysis was performed in the laboratory of the CNO.

Type 2 diabetic patients were included in this study while type 1 and type 2 diabetic with chronic eye disease such as primary open angle glaucoma, uveitis of various etiologies, having an autoimmune or neoplastic process and pregnant women were excluded. 


\section{Data collection}

During the consultation, diabetic patients who met our inclusion criteria were approached as part of our study. After obtaining their free and informed consent, patients completed the questionnaire on the survey form. Sociodemographic and anthropometric data were collected. Biological data related to diabetes were also collected from the medical record. Moreover, we performed the tear swab for the VEGF-A and Il-6 assay by ELISA. Finally, an ophthalmologic examination was carried out, where the measurement of distance visual acuity was performed; as well as an examination and photograph of the fundus after pupillary dilatation using a Topcon Fundus Camera in other to identify and classify the DR.

\section{Analysis of samples}

\section{Ophthalmological examination}

A comprehensive ophthalmological examination was performed by an ophthalmologist, focusing on the visual acuity and the fundus for DR lesions. The measurement of distance visual acuity without correction and then with correction were performed separately for each eye using the Monoyer chart. The examination of the anterior segment was carried out using a slit lamp. For the fundus, an examination associated to a retinography of the fundus was carried out after a pupillary dilatation with Tropicamide $(5 \%)$ and phenylephrine (5\%) for a detailed description of the papilla, macula and retinal vessels looking for the DR and diabetic maculopathy (DM).

Staging of DR was realized according to the International Clinical Diabetic Retinopathy Disease Severity Scale of the American Academy of Ophthalmology (AAO) (Wilkinson et al., 2003).

\section{Lacrimal swabs, protein conditioning and extraction}

Tears were collected using a Schirmer strip, placed on the edge of the eyelid at the junction of the lateral and medial thirds.
Participants did not receive prior ocular anaesthesia and kept their eyes closed during collection. The Shirmer strip was removed with gloves and placed in a $1.5 \mathrm{ml}$ centrifuge tube. The sample was immediately stored at $-20{ }^{\circ} \mathrm{C}$ until extraction and analysis (Zakaria et al., 2012; Costagliola et al., 2013).

\section{Extraction of tears}

After leaving the samples at room temperature for one hour, $50 \mu \mathrm{L} / 5 \mathrm{~mm}$ phosphate buffered saline (PBS) was introduced into the Eppendorf tube containing the Schirmer strip. The incubation step lasted for $3 \mathrm{~h}$ and eluted the tear proteins. Il- 6 and VEGF were then measured by sandwichELISA technique according to the manufacturer's instructions (Costagliola et al., 2013).

\section{ELISA analysis}

For the present study, we used the Human Il-6 Research ELISA Kit (Lot No. 8SJ2I6UYFE) and the Human VEGF-A ELISA kit (Lot No. SU5YE6RU5L) provided by Elabscience (Houston, TX, USA).

An anti-Il-6/anti-VEGF-A antibody was adsorbed on microwells. The Il-6/ VEGF present in the sample or standard bound to the antibodies adsorbed in the wells. An anti-Il6/VEGF-A monoclonal antibody conjugated to biotin was added and bound to the Il-6/VEGFA captured by the first antibody. After incubation, the unbound conjugate was removed during a wash step. Streptavidin-HRP was added and bound to the anti-Il-6/VEGF-A biotin conjugate. After incubation, the unbound Streptavidin-HRP was removed during a wash step, and the HRP-reactive Substrate Solution was added to the wells. A colored product was formed in proportion to the amount of Il-6/VEGF-A present in the sample. The reaction was stopped by addition of acid and the absorbance was measured at $450 \mathrm{~nm}$. A standard curve was prepared from seven dilutions of the Il-6/VEGF-A standard and the concentration of the Il-6/VEGF-A sample was determined (Elisa, ElabScience, USA). 


\section{Statistical analysis}

Data were recorded and analyzed using Epi Data version 7.1.5.2. and GraphPad 5.0. Results were expressed as means \pm standard deviations and percentages with $95 \%$ confidence intervals (95\% CI). Qualitative data were presented as frequencies and percentages. The differences between the groups were calculated with the Student's T-test and the nonparametric Mann-Whitney U test. Chi2 and Fischer's tests were used for comparing qualitative data. A value of $\mathrm{p}<0.05$ was considered as statistically significant.

\section{RESULTS}

A total of 81 type 2 diabetic patients were included in this study among which 34 (42\%) were men and $47(58 \%)$ women with a sex ratio of 0.72 .

\section{Prevalence of diabetic retinopathy (DR) and Diabetic maculopathy (DM)}

The prevalence of DR was found in $32.09 \%$ of cases (26 participants) while 55 $(67.90 \%)$ of patients had no DR. Among patients with DR, the number of cases with minimal DR was 13 (50\%), a moderate DR was found in $8(30.77 \%)$ cases, a severe DR in 3 (11.54\%) patients and proliferative DR in 2 (7.69\%) cases. The prevalence of diabetic maculopathy (DM) was $12.50 \%$ (10 patients). The minimal DM was found in $6(60 \%)$ cases, the moderate DM was found in $3(30 \%)$ cases, and the severe DM in $1(10 \%)$ case.

\section{Clinical data of participants}

The mean age of the participants was $56.49 \pm 10.33$ years, and the mean duration of known diabetes progression was $5.78 \pm 5.51$ years. The mean glycated hemoglobin was $8.74 \pm 2.91 \%$ and the mean blood glucose levels, $1.57 \pm 0.84 \mathrm{~g} / \mathrm{L}$. Clinical characteristics of the participants are represented in Table 1. From this table, we observed that there is a significant difference $(\mathrm{p}=0.03)$ between $\mathrm{DR}$ and non-DR group according to age. No significant difference was found with other clinical parameters.

\section{Factors associated with diabetic retinopathy}

The associations of metabolic factors with DR are presented in Table 2. Factors associated with diabetic retinopathy are high blood glucose $(\mathrm{p}=0.027)$ and glycated hemoglobin $>7 \%(\mathrm{p}=0.002)$. On the other hand, obesity and dyslipidemia are not linked to DR

In Table 3, the association between the ophthalmological factors and the DR are analyzed. From this table, DM was found in 10 participants' eyes and we observed that $\mathrm{DM}$ is significantly associated with DR ( $\mathrm{p}=0.00007)$. On the other hand, there was no association between an abnormal Schirmer test and the presence of DR ( $\mathrm{p}=0.75)$.

\section{Association between tears' cytokine profile and disease phenotype}

VEGF concentrations were detected in 30 patients, 17 and 8 of whom had DR and DM respectively. Interleukin-6 concentrations were detected in 11 patients, 06 and 3 of whom had DR and DM respectively.

The mean VEGF concentration in patients with DR was $163 \pm 45.10 \mathrm{pg} / \mathrm{mL}$; and in patients without DR was $149 \pm 81.82 \mathrm{pg} / \mathrm{mL}$ (Figure 1). The mean Il-6 concentration in patients with DR was $118.50 \pm 103 \mathrm{pg} / \mathrm{mL}$ and in patients without $\mathrm{DR}, 119 \pm 79.43 \mathrm{pg} / \mathrm{mL}$ as shown in Figure 2.

The mean VEGF concentration in patients with DM was $165.2 \pm 76.10 \mathrm{pg} / \mathrm{mL}$; and in patients without DM, it was $153.50 \pm$ $57.94 \mathrm{pg} / \mathrm{mL}$ (Figure 3). The mean Il-6 concentration in patients with DM was $54.92 \pm 32.68 \mathrm{pg} / \mathrm{mL}$ and in patients without $\mathrm{DM}, 159.10 \pm 89.78 \mathrm{pg} / \mathrm{mL}$ as shown in Figure 4. Il-6 was significantly lower in patients with $\mathrm{DM}(\mathrm{p}=0.047)$. 
Table 1: Distribution of clinical data according to diabetic retinopathy status of participants.

\begin{tabular}{lccc}
\hline & $\begin{array}{c}\text { DR } \\
(\mathbf{n = 2 6})\end{array}$ & $\begin{array}{c}\text { NDR } \\
(\mathbf{n = 5 5})\end{array}$ & \\
\hline Age (year) & Mean \pm SD & Mean \pm SD & P value \\
Duration of diabetes progression (year) & $59.81 \pm 8.84$ & $54.83 \pm 10.69$ & 0.15 \\
HbA1C (\%) & $8.43 \pm 6.23$ & $4.45 \pm 4.10$ & 0.12 \\
Blood glucose (g/L) & $9.10 \pm 2.08$ & $8.51 \pm 3.19$ & 0.32 \\
Body Mass Index & $1.76 \pm 0.87$ & $1.48 \pm 0.81$ & 0.04 \\
\hline
\end{tabular}

Table 2: Metabolic factors associated with diabetic retinopathy status.

\begin{tabular}{|c|c|c|c|c|}
\hline & DR & NDR & OR (IC 95\%) & *P Value \\
\hline \multicolumn{5}{|l|}{ Blood glucose (g/L) } \\
\hline$>1.25$ & 17 & 20 & 2.89 (1.11-7.52) & 0.027 \\
\hline$<1.25$ & 10 & 34 & & \\
\hline \multicolumn{5}{|l|}{ HbA1C (\%) } \\
\hline$>7$ & 20 & 21 & $4.49(1.62-12.45)$ & 0.002 \\
\hline$<7$ & 7 & 33 & & \\
\hline \multicolumn{5}{|l|}{ Obesity (BMI) } \\
\hline$>30$ & 9 & 18 & & 1000 \\
\hline$<30$ & 18 & 36 & $1.00(0,38-2,66)$ & 1.000 \\
\hline \multicolumn{5}{|l|}{ Dyslipidemia } \\
\hline yes & 7 & 13 & $130(044382)$ & 0634 \\
\hline No & 17 & 41 & $1.30(0.44-3.82)$ & 0.034 \\
\hline
\end{tabular}

BMI: Body mass index

DR: diabetic retinopathy

NDR: non diabetic retinopathy

* $p$ value: obtained by Chi square and Fisher's test

Table 3: Ophthalmological factors associated with diabetic retinopathy status.

\begin{tabular}{rcccc}
\hline & DR & NDR & OR (IC 95\%) & P value \\
\hline Diabetic maculopathy & & & & \\
Yes & 9 & 1 & $\mathbf{3 0 . 3 8}(\mathbf{3 . 5 7 - 2 5 8 . 1 8})$ & $\mathbf{0 . 0 0 0 0 7}$ \\
No & 16 & 54 & & \\
Schirmer Test $(\mathrm{mm})$ & & & & 0.75 \\
$<15$ & 12 & 26 & $0.86(0.34-2.18)$ & \\
$>15$ & 15 & 28 & &
\end{tabular}

DR: diabetic retinopathy

NDR: non diabetic retinopathy

* p value :obtained by Chi square and Fisher's test 


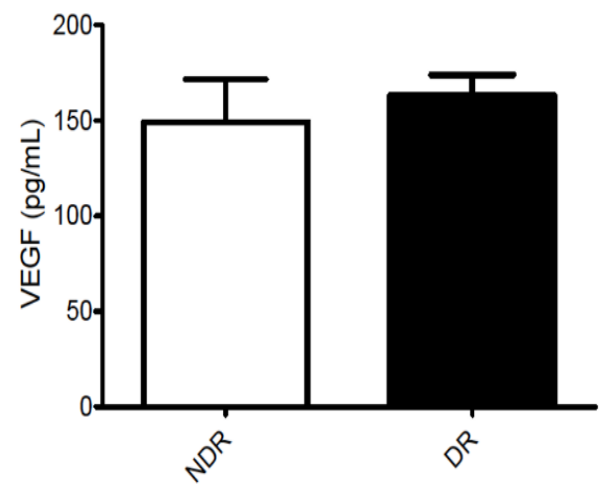

DR: diabetic retinopathy

NDR: non diabetic retinopathy

Data are expressed in mean \pm Standard deviation

$P$ value: 0.2929 (Mann-Whitney test)

Figure 1: Analysis of lacrimal VEGF-A according to the presence of diabetic retinopathy.



DR: diabetic retinopathy

NDR: non diabetic retinopathy

Data are expressed in mean \pm Standard deviation

P value: 0.4971 (Mann-Whitney test)

Figure 2: Analysis of lacrimal Il-6 according to the presence of diabetic retinopathy.
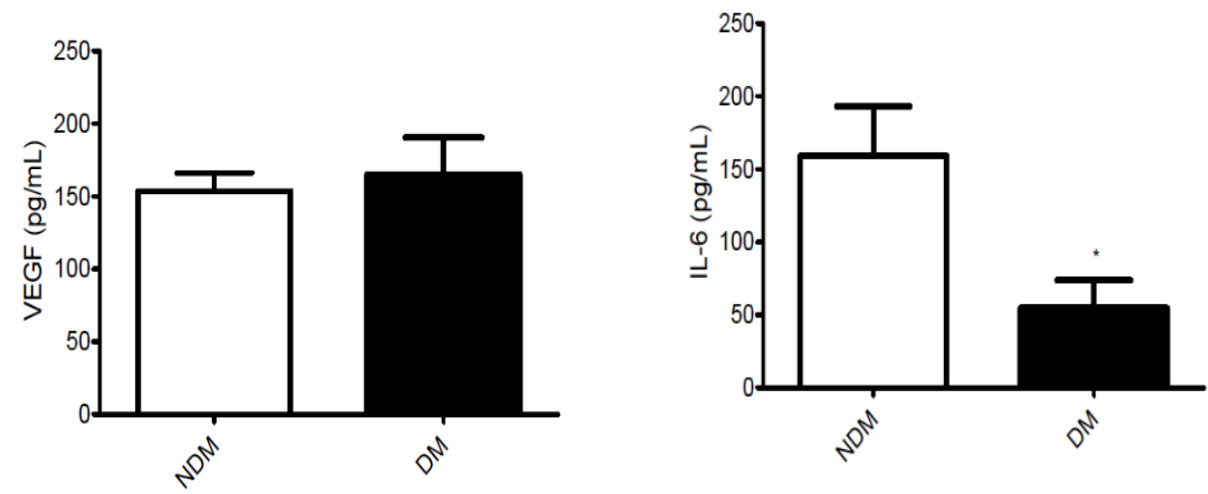

DM: diabetic retinopathy

NDM: non diabetic retinopathy

Data are expressed in mean + Standard deviation

P value: 0.3437 (Mann-Whitney test)

Figure 3: Analysis of lacrimal VEGF-A according to the presence of diabetic maculopathy

DM: diabetic retinopathy

NDM: non diabetic retinopathy

Data are expressed in mean + Standard deviation

P value: 0.047 (Mann-Whitney test)

Figure 4: Analysis of lacrimal Il-6 according to the presence of diabetic maculopathy 


\section{DISCUSSION}

The aim of the present study was to compare two lacrimal angiogenic mediators of diabetic retinopathy in diabetic patients followed at the Yaoundé Central Hospital. Proteomic analysis can be an innovative means to bring information on the state of the disease but also on the treatment response (Und Hohenstein-Blaul et al., 2013).

Diabetic retinopathy and diabetic maculopathy were found in $32.10 \%$ and $12.50 \%$ of patients respectively. The prevalence of DR obtained is superposable to that of Jingi et al. (2014) in Cameroon who reported a prevalence of DR at $40.3 \%$ and that of the DM at $14.5 \%$ (2014). There was an association between the DR and the DM $(p=0.0002)$. Indeed, the DM is very often associated with DR (Ciulla et al., 2003) and characterized by the accumulation of extracellular fluid in Henle's layer and the inner nuclear layer of the retina. The extravasation of fluid is explained by the mechanism of the breakdown of the blood retinal barriers where VEGF plays an important role (Antcliff and Marshall, 1999).

The ELISA technique was used for the detection of biomarkers in the tear samples according to the protocol described by Costagliola (2013) in the present study (2013). VEGF-A concentration in tears of patients with DR was superposable compared to those without DR $(p=0.2929)$. Moreover, no significant difference was found for lacrimal concentration of Il- 6 between patients with DR and those without $(\mathrm{p}=0.4971)$. However, the level of Il-6 in tears amongst patients with DM was significantly lower compared to those without DM. VEGF-A has been described to be involved in blood-retina barrier injury and neovascularization process (Funatsu et al., 2001). According to the literature, VEGF and Il-6 concentrations analyzed in intraocular fluids are related to the presence of DR. They are also related to the progression of DR and the level of cytokines increased in the proliferative form of DR (Funatsu et al., 2005; Koleva- Georgieva et al., 2011; Dong et al., 2013). Moreover, some studies showed that IL6 concentration is higher in the vitreous of patients with DM and that its level is significantly correlated to that of VEGF (Funatsu et al., 2003). The high level of these cytokines in intraocular fluids is due to their ability to enhance the vascular permeability and participate to the stimulation of the neovascularization in the eyes of diabetic patients (Mesquida et al., 2019). According to the literature, tear proangiogenic cytokines are more represented in the eyes of diabetic patients (Liu et al., 2010; Ang et al., 2019). Ang et al. (2019) in their study on the analysis of tear VEGF in diabetic patients reported that the level of this biomarker in tears is higher amongst patients with DR compared to those without DR (Ang et al., 2019). However, the results obtained in the present study are different compared to those reported by Ang et al. (Ang et al., 2019). The justification of our results may be the site of production of these biomarkers. As reported by Amin (1997), VEGF is present in the retina of diabetic patients with no or little retinal vascular abnormality (1997). The expression of VEGF therefore precedes the retinal neovascularization (Amin et al., 1997; Boulton et al., 1998). Moreover, a mechanism of sequestration can justify our results for the level of VEGF-A in tears, because this biomarker is produced in large quantities by the retina, it is confined in the posterior segment of the globe with no or little difference in different groups of diabetic patients with and without retinopathy.

Il-6 is a proinflammatory cytokine synthetized by several types of cells including lymphocytes, macrophages, retinal pigment epithelium (Kishimoto, 1989). Its increase in ocular fluids reflects an ocular inflammation and is associated with the disruption of the blood-retinal barrier (Funatsu et al., 2003). The results concerning Il-6 in our work can be due to the fact that inflammation process is located in the posterior segment (Mocan et al., 2004). Nevertheless, it could also be explained by the size of the sample.

Tear film abnormality was not associated with diabetic retinopathy. These findings are close to those conducted by Zhang and Leiden (Van Leiden et al., 2002; Zhang et 
al., 2018). However, a correlation between tear film abnormality and DR has also been described in the literature (Nepp et al., 2000; Kesarwani et al., 2017).

Early detection of DR and its treatment decrease the apparition and progression of complications. In this study, we found that minimal and moderate DRs were the most prevalent forms. Koki et al. (2010) and Jingi et al. (2014) found a different distribution of the stages of DR where the proliferative form was most frequent. These differences may be due to the location of the patients' recruitment in an unit of ophthalmology where patients are referred especially when there is a visual acuity disturbance.

Metabolic factors associated with DR were glycated hemoglobin $>7 \%$ and fasting blood glucose > 1.25g/L. Imbalance of glycated hemoglobin in a treated diabetic patient reflects poorly controlled diabetes, resulting in microvascular complications. These results are consistent with others studies conducted among diabetic patients (Dermitas et al., 2015; Noche et al., 2020). Obesity and dyslipidemia were not associated with DR; indeed, a meta-analysis study by Zhou et al. (2018), showed that high body Mass Index (BMI) doesn't increase the risk of DR (Zhou et al., 2018). However, since overweight and obesity are important cardiovascular risk factors, it is still imperative to maintain an optimal BMI.

\section{Limits}

To the best of our knowledge, this is one pilot study showing the possible role of tears in the detection of diabetic retinopathy. However, this method needs further studies on larger samples with different biomarkers for the development of a tool that could be used to screen diabetic patients.

\section{Conclusion}

The present study showed that lacrimal concentration of VEGF-A and Il-6 was not related to the presence of diabetic retinopathy. However, the level of Il-6 in tears was associated with the diabetic maculopathy. This method of investigation of biomarkers may be useful for the development of tools for the diagnosis in diabetes and diabetic retinopathy.

\section{COMPETING INTERESTS}

The authors declare that they have no competing interests.

\section{AUTHORS' CONTRIBUTIONS}

DC, CD, SE made a substantial contribution to the design of the work. DC, $\mathrm{CD}$, SNF, NLA, GTB, NFU and SE contribute to the acquisition, analysis or interpretation of data. All authors brought a contribution to the elaboration of the manuscript and revised it critically for important intellectual content, and approved to be published.

\section{ACKNOWLEDGEMENTS}

We thank the personnel of the National Obesity Centre (CNO) of the Yaounde's Central Hospital and Innel Medical Center for their help in patient recruitment and sample collection.

\section{REFERENCES}

Amin RH, Franck RN, Kennedy A, Elliot D, Puklin JE, Abrams GW. 1997. Vascular endothelial growth factor is present in glial cells of the retina and optic nerve of human subjects with non proliferative diabetic retinopathy. Investigative Ophthalmology \& Visual Science, 38(1): 36-47.

Ang WJ, Zunaina E, Norfadzillah AJ, RajaNorliza RO, Julieana M, Ab-Hamid SA, Mahaneem M. 2019. Evaluation of vascular endothelial growth factor levels in tears and serum among diabetic patients. PloS ONE, 14(8): e0221481. DOI: $10.1371 /$ journal.pone. 0221481

Antcliff RJ, Marshall J. 1999. The pathogenesis of edema in diabetic maculopathy. Seminars in Ophthalmology, 14(4): 223-32. DOI: 10.3109/08820539909069541

Boulton M, Foreman D, Williams G, McLeod D. 1998. VEGF localisation in diabetic retinopathy. British Journal of Ophthalmology, 82(5):561-568. DOI: 10.1136/bjo.82.5.561 
Burgess PI, Msukwa G, Beare NA. 2003. Diabetic retinopathy in sub-Saharan Africa: meeting the challenges of an emerging epidemic. BMC Medicine, 11(1):157. DOI: $10.1186 / 1741-7015-11-$ 157

Cheung N, Mitchell P, Wong TY. 2010. Diabetic Retinopathy. Lancet, $\mathbf{3 7 6}$ (9735): 124-36. DOI: 10.1016/S01406736(09)62124-3

Ciulla TA, Amador AG, Zinman B. 2003. Diabetic retinopathy and diabetic macular edema: pathophysiology, screening, and novel therapies. Diabetes Care, 26(9): 2653-2664.

DOI: 10.2337/diacare.26.9.2653

Costagliola C, Romano V, De Tollis M, Aceto F, dell'Omo R, Romano MR, Pedicino C, Semeraro F. 2013. TNF-Alpha Levels in Tears: A Novel Biomarker to Assess the Degree of Diabetic Retinopathy. Mediators of Inflammation, 626529. DOI: 10.1155/2013/629529

Demirtas L, Degirmenci H, Akbas EM, Ozcicek A, Timuroglu A, Gurel A, Ozcicek F. 2015. Association of hematological indicies with diabetes, impaired glucose regulation and microvascular complications of diabetes. International Journal of Clinical and Experimental Medicine, 8(7):1142011427.

Dong N, Xu B, Wang B, Chu L. 2013. tudy of 27 aqueous humor cytokines in patients with type 2 diabetes with or without retinopathy. Molecular Vision, 19:173446.

Ebneter A and Zinkernagel M. 2016. Novelties in Diabetic Retinopathy. Novelties in Diabetes, 31: 84-96. DOI:10.1159/000439391

Funatsu H, Yamashita H, Shimizu E, Kojima R, Hori S. 2001. Relationship between vascular endothelial growth factor and interleukin-6 in diabetic retinopathy. Retina, 21(5): 469-77. DOI: 10.1097/00006982-2001100000-00009

Funatsu H, Yamashita H, Ikeda T, Mimura T, Eguchi S, Hori S. 2003. Vitreous levels of interleukin-6 and vascular endothelial growth factor are related to diabetic macular edema. Ophthalmology, 110(9):1690-6. DOI: $10.1016 / \mathrm{S} 061$ 6420(03)00568-2

Funatsu H, Yamashita H, Noma H, Mimura T, Nakamura S, Sakata K, Hori S. 2005. Aqueous humor levels of cytokines are related to vitreous levels and progression of diabetic retinopathy in diabetic patients. Graefe's Archive for Clinical and Experimental Ophthalmology, 243(1): 3-8. DOI: 10.1007/s00417-0040950-7

Ghasemi H. 2018. Roles of Il-6 in ocular inflammation: a review. Ocular Immunology and Inflammation, 26(1): 37-50.

DOI:

10.1080/09273948.2016.1277247

Hagan S, Martin E, Enríquez-de-Salamanca A. 2016. Tear Fluid Biomarkers in Ocular and Systemic Disease: Potential Use for Predictive, Preventive and Personalised Medicine. EPMA Journal, 7(1): 15. DOI: 10.1186/s13167-016-0065-3

Jingi A M, Noubiap JJN, Ellong A, Bigna JJR, Mvogo CE. 2014. Epidemiology and Treatment Outcomes of Diabetic Retinopathy in a Diabetic Population from Cameroon. BMC Ophthalmology, 14: 19. DOI: $10.1186 / 1471-2415-14-19$

Kesarwani D, Rizvi SWA, Khan AA, Amitava AK, Vasenwala SM, Siddiqui Z. 2017. Tear film and ocular surface dysfunction in diabetes mellitus in an Indian population. Indian J Ophthalmol, 65(4): 301-304. DOI:10.4103/ijo.IJO_939_15

Kishimoto K. The biology of Interleukin 6 . 1989. Blood, 74: 1-10.

Koki G, Bella AL, Omgbwa EA, Epee E. 2010. Rétinopathie diabétique du Noir africain: étude angiographique. Cahiers de Santé, 20(3): $\quad 127-132 . \quad$ DOI: 10.1684/san.2010.0207

Koleva-Georgieva DN, Sivkova NP, Terzieva D. 2011. Serum inflammatory cytokines IL-1 $\beta$, IL-6, TNF- $\alpha$ and VEGF have influence on the development of diabetic retinopathy. Folia Medica, 53(2): 44-50. DOI: 10.2478/v10153-010-0036-8 
Labie D. 2007. Le diabète en Afrique subsaharienne. Médecine/Sciences, 23(3): 320-322.

Liu J, Shi B, He S, Yao X, Wilcox MD, Zhao Z. 2010. Changes to Tear Cytokines of Type 2 Diabetic Patients with or without Retinopathy. Molecular Vision, 16: 2931-2938.

Mesquida M, Drawnel F, Fauser S. 2019. The role of inflammation in diabetic eye disease. Seminars in Immunopathology; 1-19. Springer Berlin Heidelberg. DOI: 10.1007/s00281-019-00750-7

Mocan MC, Kadayifcilar S, Eldem B.2006. Elevated intravitreal interleukin-6 levels in patients with proliferative diabetic retinopathy. Canadian Journal of Ophthalmology, 41(6): 747-52. DOI: 10.3129/106-070

Myśliwiec M, Balcerska A, Zorena K, Mysliwska J, Lipowski P, Raczynsa K. 2008. The Role of Vascular Endothelial Growth Factor, Tumor Necrosis Factor Alpha and Interleukin-6 in Pathogenesis of Diabetic Retinopathy. Diabetes Research and Clinical Practice, 79(1): 141-146.

DOI: 10.1016/j.diabres.2007.07.011

Nepp J, Abela C, Polzer I, Derbolav A, Wedrich A. 2000. Is there a correlation between the severity of diabetic retinopathy and keratoconjunctivitis sicca? Cornea, 19(4): 487-491.

Noche CD, Kouosseu FN, Bilong Y, Djamen PC, Kwetche PF, Lowe AN, Agoons B, Temgoua M, Katte Djabou JC, Sobgwi E. 2020. Relationship between Angiogenic and Inflammatory Biomarkers and Diabetic Retinopathy. Open Journal of Ophthalmology, $\mathbf{1 0}(01)$ : $44 . \quad$ DOI: 10.4236/ojoph.2020.101006

OMS. 2020. OMS | Maladies Oculaires Prioritaires. WHO. https://www.who.int/blindness/causes/pri ority/fr/index 1.html
Reutrakul S, Crowley SJ, Park JC, Chau FY, Priyadarshini M, Hanlon EC, Danielson KK, Gerber BS, Baynard S, Yeh JJ, McAnany JJ. 2020. Relationship between Intrinsically Photosensitive Ganglion Cell Function and Circadian Regulation in Diabetic Retinopathy. Scientific Reports, 10(1): 1-11. DOI: 10.1038/s41598-020-58205-1

Tarr JM, Kaul K, Wolanska K, Kohner EM, Chibber R. 2012. Retinopathy in diabetes. Advances in Experimental Medicine and Biology, 771: 88-106. DOI: 10.1007/9781-4614-5441-0_10

Und Hohenstein-Blaul NV, Funke S, Grus FH. 2013. Tears as a source of biomarkers for ocular and systemic diseases. Experimental Eye Research, 117: 126-37. DOI: 10.1016/j.exer.2013.07.015

Van Leiden HA, Dekker JM, Moll AC, Nijpels G, Heine RJ, Bouter LM, Stehouwer C, Polak B. 2002. Blood Pressure, Lipids, and Obesity Are Associated with Retinopathy: The Hoorn Study. Diabetes Care, 25(8): 1320-1325. DOI: 10.2337/diacare.25.8.1320

Zakaria N, Van Grasdorff S, Wouters K, Rozema J, Koppen C, Lion E, Cools N, Berneman Z, Tassignon MJ. 2012. Human Tears Reveal Insights into Corneal Neovascularization. PloS ONE, 7(5): e36451. DOI: 10.1371/journal.pone.0036451

Zhang, J, Wang Y, Li L, Zhang R, Guo R, Li H. 2018. Diabetic Retinopathy May Predict the Renal Outcomes of Patients with Diabetic Nephropathy. Renal Failure, 40(1): 243-251. DOI: 10.1080/0886022X.2018.1456453

Zhou Y, Wang C, Shi K, Yin X. 2018. Relationship between Dyslipidemia and Diabetic Retinopathy. Medicine, 97(36): e12283. DOI: 10.1097/ MD. 0000000000012283. 